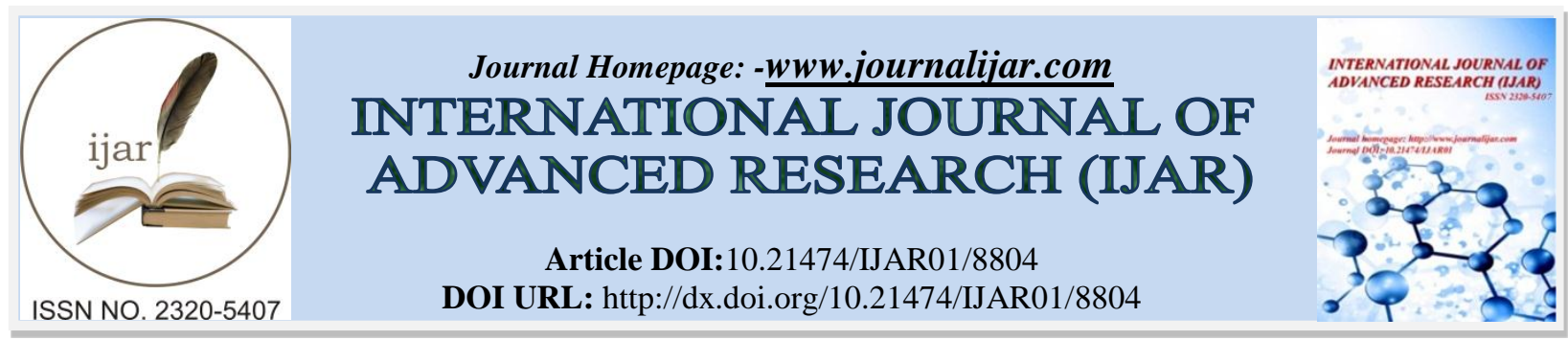

RESEARCH ARTICLE

\title{
STUDY OF GENE-ENVIRONMENT CAUSES OF MAJOR DEPRESSIVE DISORDER TO STATISTICALLY DEFINE THE MOST COMMON CAUSE BEHIND IT.
}

\author{
Raktima Sengupta ${ }^{1}$, Sampurna $\operatorname{Roy}^{2}$ and Moinak Bhattacharya ${ }^{3}$. \\ 1. B.Tech, Department of Biotechnology(Genetics), SRM Institute of Science and Technology, Kattankulathur. \\ 2. B.Tech, Department of Computer Science and Engineering(Cyber Security), SRM Institute of Science and \\ Technology, Kattankulathur. \\ 3. B.Tech, Department of Computer Science and Engineering SRM Institute of Science and Technology, \\ Kattankulathur.
}

\section{Manuscript Info}

[.......................

Manuscript History

Received: 04 February 2019

Final Accepted: 06 March 2019

Published: April 2019

Key words:-

neuro-biological,etiological,psychobiological, gene hunting, chronicstress.

\section{Abstract}

Depression (Major Depressive Disorder) is a common and serious medicalillness that negatively affects how one feels, the way one thinks and how one acts. Depression causes feelings of sadness and/or a loss of zest in doing activities once enjoyed by a victim. It can lead to a variety of emotional and physical problems and can decrease a person's productivity and efficiency at work and at home thereby bringing one's mental and physical health down. Environmental factors play a very prominent role in causing depression apart from neuro-biological and genetic factors. There are a number of factors such as pollution, childhood abuse, long term stress, traumatic events that could lead to Major Depressive Disorder(MDD). There are also "depression genes". Some diseases are caused by single defective gene such as cystic fibrosis or Huntington's disease. However depression is also influenced by several genes rather than caused by a single gene. In these conditions there are combinations of genetic changes that can cause depression. Nevertheless the etiological and psycho-biological complexity of depression makes it difficult for identifying the specific genetic factors and hence it continuous to be among the last frontiers of gene hunting. Some other queer factors leading to depression are Oedipus Complex, Genetic Sexual Attraction (GSA), and having different sexual orientation(LGBTQ). In this paper the authors first try to explore the various causes of depression using a database of patients suffering from clinical depression. Depression is a vast arena. We as young researchers have tried to narrow down our focus mainly on patients suffering from clinical depression; trying to figure out the most common reason behind it. Our survey therefore focuses on genetic and environmental (post-rape trauma, chronic stress, childhood abuse, allergy) causes of depression. Our purpose is to give a better understanding to the cause of depression to the patients so that they can avoid it. Thereby the paper tries to statistically define the most prominent cause of Major Depressive Disorder. 


\section{Introduction:-}

Depression is a major disorder of mood characterized by a persistent dysphoria, anxiety, irritability, fear, brooding, appetite and sleep disturbances, weight loss, psychomotor agitation or retardation, decreased energy, feelings of worthlessness or guilt, difficulty in concentrating or thinking, possible delusions and hallucinations, and thoughts of death or suicide. The disorder, which occurs in children, adolescents, and adults, may develop over a period of days, weeks, or months. We call it the four step viscous cycle of Incidence, Prevalence, Mortality and Lifetime risk. The reasons could be many specifically categorizing them into environmental, psychological and genetic causes. Episodes may occur in clusters or singly, separated by several years of normality. The causes of the disorder are multiple and complex and may involve biological, psychological, interpersonal, and sociocultural factors that lead to an unidentifiable intra psychic conflict. Treatment includes use of antidepressants like FETZIMA and electroconvulsive therapy, followed by long-term psychotherapy. Nursing care is needed to ensure adequate nutrition, appropriate balance of fluid intake and output, good personal hygiene, and protection of the patient from self-injury. It is also called major affective disorder or unipolar disorder. Genetically triggered determinants leading to Major Depressive Disorders include diseases like Dysthymia, Bipolar Disorder, Schizophrenia, Schizoaffective Disorder, etc. Environmental changes play a major role in bringing psychological changes in a person thereby leading to various kinds of changes in their attitude which might take the shape of Major Depressive Disorder. All of these various environmental, psychological and genetic factors which give rise to depression shall be further discussed in the paper.

\section{Role of genetics in mdd[10,11,13]:}

Many times it has been asked that is there any genetic analysis of Major Depressive Disorder or does depression triggers because of some genetic factors too; the answer is yes. It is a matter of great interest among the scientists to know what are the different genes inside a human body which can trigger Major Depressive Disorder. Studies show that there are almost $60 \%-65 \%$ [32] people in the world who are suffering from depression right now in which the level of depression varies according to person to person. In this paper, we have focused about the genetically influenced diseases which eventually roots to depression and amount of people suffering from such disorders which leads them to Major Depressive Disorder. The study of different diseases concluded with a record of some major disorders which widely affects most of the people in India. It includes:

Dysthymia,Bipolar Disorders, (c)Schizophrenia, (d)Schizoaffective Disorders, (e)Autism Spectrum Disorder and

(f) Huntington's Disease.

\section{Dysthymia (Persistent Depressive Disorder):}

Just like other types of depression, Persistent Depressive Disorder causes some kind of deep and continuous sadness and hopelessness in a person during their daily routine. These feelings can affect a person's behavior, mood and as well as physical functions which also includes appetite and sleep. As a result, people with this disorder often lose their interest in doing activities they once used to enjoy and face some difficulty in finishing daily tasks. During the study of Dysthymia it has been found that this disease can occur because of some genetic trait and can be inherit from blood relatives. There are four CRY2 genetic variants (rs10838524, rs7121611, rs7945565, rs1401419) [1] which are significantly associated with dysthymia. Researches [21] have shown that the single nucleotide polymorphisms (SNPs) in the genes CACNA1C, ODZ4, and NCAN, have emerged as promising candidate genes for bipolar disorder in genome-wide association studies. This kind of disorder is mostly found among the people having age of 19 years or above.

\section{Bipolar disorder:}

Bipolar disorder, also known as manic depression or maniac depressive disorder, is a kind of mental illness that brings severe low and high moods and changes in sleep, energy, thinking, and behavior. It is widely believed that the foundations 
bipolar are to be largely genetic, which happens to be the strongest and most consistent threat point for the disease (APA, 2013). We have found it to be occurring between the ages of 14 to $60 \mathrm{yrs}$. As the bipolar disorder is passed through family generational lines, it appears to affect how the brain is formed to handle mood regulation and its other symptoms. Studies have found some genes that may play a major role behind Bipolar Disorder which includes DRD1, DRD4, DAT1, HTTLPR, HTR2A, BDNF, and NCAM 1(and possibly many more).[22] (c)[2] Schizophrenia:

This kind of disorder or disease is mostly observed in people above the age of 14 yrs. Schizophrenia is a serious mental illness that interferes with a person's ability to think clearly, manage their emotions, make decisions and relate to others and thus end up getting highly depressed. This is a complex, long-term medical illness; many different genes may increase the risk of schizophrenia, but that no single gene causes the disorder by itself. Well, new genome scan project, provide support for schizophrenia candidate region on chromosome 1q, 2q, 5q, 6p, 8p, 10p, $13 q, 15 q$ and $22 q[23]$. It is not yet possible to use genetic information to predict who will develop schizophrenia as there are many genes which can possibly trigger this disease. Although, recent studies on molecular genetics of schizophrenia which focused on positional and functional candidate genes postulated to be associated with schizophrenia has discovered genes such as neuregulin (NRG-1, 8p12-21), dysbindin, (DTNBP1,6p22.3), G72 (13q34) / D-amino acid oxidase (DAAO,12q24), proline dehydrogenase (PRODH-2, 22q11.21), catechol-Omethyltransferase(COMT, 22q11.21), regulator of G protein signaling (RGS-4), 5HT2A and dopamine D3 receptor (DRD3) which happens to be some of those many genes that can cause such disorder. [25] (d) Schizoaffective Disorder:

Schizoaffective Disorder is certainly a combination of various disorders such as schizophrenia, mood disorders and bipolar disorder. It is observed mainly above the age of 19 yrs. There are studies which suggest that variations in many genes, each with a small effect, combine to increase the risk of developing this condition. There may also be genetic variations with larger effects in some affected families or individuals, but these types of variants are rare in the general population, and it is still unclear that which particular genes are involved in this process. There are two types of Schizoaffective Disorder and each has some schizophrenia symptoms in it-

1. Bipolar: It includes episodes of mania and sometimes major depression.

2. Depressive: This has only major depressive episodes.

Autism Spectrum Disorder (Asperger's syndrome, pervasive developmental disorder): Autism spectrum disorder (ASD) is a developmental disorder that affects communication and behavior of a person. Although it can be diagnosed at any age, it is believed to be a "developmental disorder" because symptoms generally appear in the first two years of life of a person. Finding all the causes of Autism Spectrum Disorders is still a big topic for the researchers. Scientists know that genetics are one of the major risk factors in this kind of disorder. But they don't have all of the answers yet. There is not any one "autism gene" that happens to be at work. In studying, data from whole-genome screens in

multiplex families suggest interactions of at least 10 genes that can cause autism. Thus far, a presumed speech and language region at 7q31-q33 seems most dynamically linked to autism. Among other candidate genes are the FOXP2, RAY1/ST7, IMMP2L, and RELN genes at 7q22-q33 and the GABA(A) receptor subunit and UBE3A genes on chromosome 15q11-q13. Variant alleles of the serotonin transporter gene (5-HTT) on 17q11-q12 [24] are more frequent in individuals with autism than in non-autistic populations. Certainly many other things too, in addition to the genes, may be involved in other ways. Autism spectrum disorders leads to a Major Depressive Disorder at some point of the victim's life as for the person it includes social, communication, and behavioral challenges during the period of their disorder. These problems can be mild, severe, or somewhere in between. It happens mostly to the children from early childhood; from 9 to 12 months of age.

\section{Huntington's disease:}

Huntington's disease is a fatal genetic disorder that generates the progressive breakdown of nerve cells in the brain of a person. It deteriorates a person's physical and mental abilities during their prime working years and has no cure and ultimately leads to depression. HD is known as the quintessential family disease because every child of a parent with this disease has a 50/50 chance of carrying the defective gene. The symptoms of this disease usually appear between the ages of 30 to $50 \mathrm{yrs}$., and gets worse over a 10 to 25 year period. Ultimately, the victim succumbs to pneumonia, heart failure or other complications in their life. Everyone has the gene that causes Huntington's disease, but only those that inherit the expansion of the gene will somehow develop this and perhaps pass it on to each of their children. The genetic defect in the "HD gene", which occurs to be the main gene behind the cause of Huntington's disease, explains many of the genetic features of the disorder. This gene is located on the chromosome $4 \mathrm{p} 16.3$ 
[4].This shows that the genetic basis of Depressive Disorders overlaps very predominantly with other psychiatric disorders like bipolar disorder and schizophrenia etc. From the above list of diseases or disorders it is specifically cleared that there are many disorders which give rise to Major Depressive Disorders because of involvement or we can say mutation of genes. Depression is a vast field for research and there are certainly many other genetic disorders which eventually construct a pathway for depression in a person's life and yet have not been discovered. During early researches it was claimed that there a "depression gene" which happens to be the reason of depression in an individual but as the researches increased, some scientists conclude that there is no any single gene for depressive disorders. There are many other genes which unquestionably somehow hike MajorDepressive Disorder.

\section{The Concept Of Depression Gene [6][7][8]}

A British research team [26] has recently isolated a gene which appears to be prevalent in multiple family members Depressive Disorders. In this research, the chromosome named 3p25-26 was found in more than 800 families with recurrent depression. Scientists also believe that as many as 40 percent of those with depression can trace it to a genetic link. Environmental and other factors make up the other 60 percent. There is a report in The Independent that "Scientists have for the first time established a genetic cause for depression narrowing it down to a specific chromosome". The Independent said "the study has found 'clear evidence' that a region on chromosome 3 (called 3 p25-26) is linked to severe recurrent depression". There were 214 genes within the region of chromosome 3 identified as being linked to severe recurrent depression.

There is another report in the journal Biological Psychiatry, in which researchers from Erasmus University Medical Centre in The Netherlands published a new study. They identified one gene implicated in Major Depressive Disorder called NKPD1. The researchers took advantage of a genetically homogeneous group of people called the Erasmus Rucphen Family (ERF) which is a cohort of 3000 people who are all scion of 22 couples from 18th century [27]. The group lives in an isolated village in the Netherlands and has had very few people join from the outside, resulting in a restricted genetic population. When given a self-reported test, 2,353 of them showed the symptoms of depression. Further, this study also found an association between NKPD1 and people outside of the ERF, when they looked in the general population.

So conclusively there is no any specific gene inside a human body which can be called as a "depression gene" which technically shows that the concept of specific depression gene is certainly inaccurate. There are still many hidden genes and chromosomes which are playing there vital role in rooting depression. There are many researchers who believe that it is not a singular gene that get someone into the risk of mental illness. It is more likely a combination of genes or genome that guides to the Major Depressive Disorder. Thus, the causes of bipolar disorder, anxiety disorders and other disorders/diseases may also be traced to a combination of genetic factors. Research has also shown that people with parents or siblings who have depression are up to three times more likely to have the condition. This can be due to heredity or environmental factors that have a strong influence.

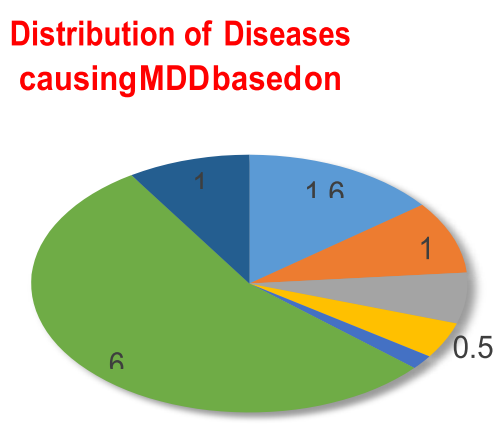

- Dysthymia

Bipolar Disorder

- Schizoaffective Disorder

- Schizophrenia

Other Genetical Disorders

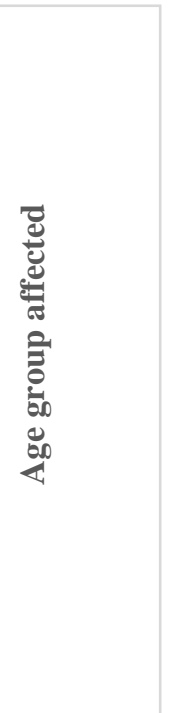

Figure 1:-Distribution of Diseases causing îī̄ĩ based on generaiized popuiation experimented on 3 randomly distributed samples and sample data. 


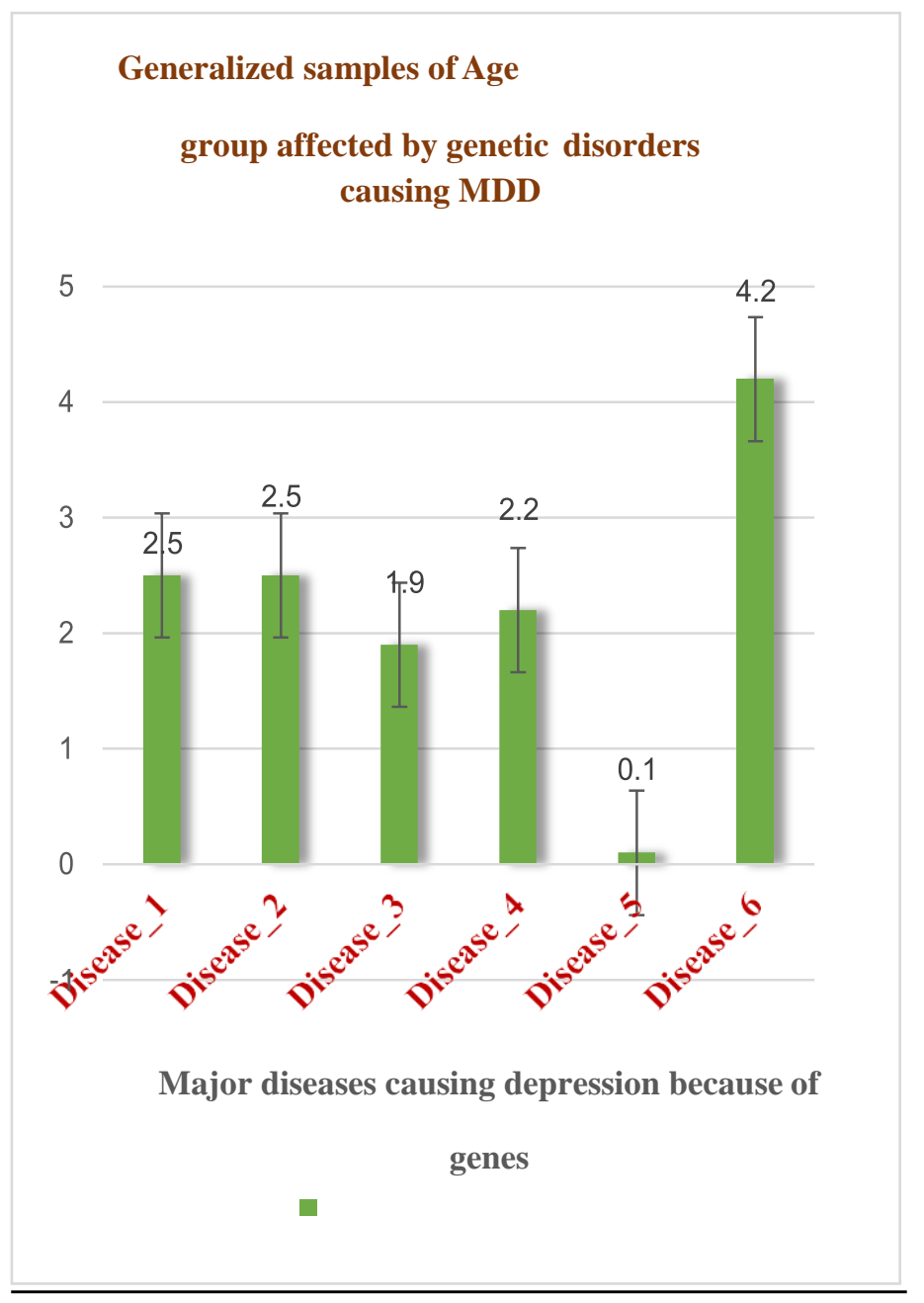

\section{Personality traits:}

Several scientists like Carl Jung [28], Sigmund Freud [33] and Erik Erikson [34] and many more since the advent of time have classified human personalities into categories taking their classification into consideration we have studied the several types of personalities people have. From the survey conducted, the weaknesses of the several personality types have been identified which gave clear indications of symptoms of Major Depressive Disorder. A needy attitude or feeling too introverted all the time can lead to depression. Unrelenting imaginative personality types lead to disappointments very easily which if gets a regular thing leads to frustration. Chronic frustration gives rise to stress both of which are clear symptoms of a person gradually falling victim to depression. Certain personality types have a trait of anti-pathy to rules and tendency overanalyze and be judgmental, even arrogant. All of this adds up to a personality type which is often clueless in dating and other environmental and sociological interactions which again lead frustration over a period of time resulting in depression. Over analytical personality types often tend to suffer from dis-satisfaction thereby suffering from clinical depression. Condescending is another major reason behind depression. Certain type of characteristic traits personality types attempt at connecting with others which is often worse than their withdrawal. These type of people take pride in their knowledge and rationalize and enjoy sharing their ideas but in trying to explain how they got from A to B to $\mathrm{Z}$, they can get sometimes simplifying things to the point of insult as they struggle to gauge their conversation partners' perspective. The ultimate insult comes as they give up with a dismissive "never mind". If this happens on a regular basis which often does then the chronic frustration lead to depression. Certain personality types are over empathetic which can also be a reason for them suffering from clinical depression. Self-loathing (very strong feelings of dislike for themselves) is another weakness many can't fight and thereby fall victim to depression. Another cause behind depression is being too altruistic. These types of people sometimes see themselves as selfish, but only because they want to give so much more than they are 
able to. This becomes a self-fulfilling prophecy, as they try to push themselves to commit to a chosen cause or person, forgetting to take care of the needs of others in their lives, especially themselves which can later lead to midlife crisis which is a prominent cause of Major Depressive Disorder. Extremely condemning nature can to a person being highly depressive in nature. Certain personality types repress their feelings. They internalize their feelings a great deal. This lack of healthy emotional expression can lead to a lot of stress and frustration thereby leading to dejection and pessimistic behavior. Certain personality types suffer from immense insecurities (insecurities of all kinds) and if this happens for a prolonged period of time it can lead to clinical depression. Few personality have been detected to suffer from Hypochondria which is quite a prominent symptom of MDD.

\section{Psycho-environmental factors [9]: \\ Obsessive Compulsive Disorder(OCD) $[14,15]$ :}

Obsessive-Compulsive Disorder (OCD) [29] is a common, chronic and long-lasting disorder in which a person has uncontrollable, reoccurring the Obsessions) and behaviors (compulsions) that he or she feels the urge to repeat over and over. People with OCD may have symptoms of obsessions, compulsions, or both. These symptoms can interfere with all aspects of life, such as work, school, and personal relationships. Obsessions may also include Fear of germs or contamination, unwanted forbidden or taboo thoughts involving sex, religion, and harm, aggressive thoughts towards others or self, having things symmetrical or in a perfect order.

Compulsions are repetitive behaviors that include: Excessive cleaning and/or hand-washing, Ordering and arranging things in a particular, precise way, Repeatedly checking on things, such as repeatedly checking to see if the door is locked or that the oven is off, Compulsive counting. People with OCD can't control his or her thoughts or behaviors, even when those thoughts or behaviors are recognized as excessive, spends at least 1 hour a day on these thoughts or behaviors, doesn't get pleasure when performing the behaviors or rituals, but may feel brief relief from the anxiety the thoughts cause, experiences significant problems in their daily life due to these thoughts or behaviors. OCD is a disease that should be treated else it starts interfering with all aspects of life, depression being the most common disease that people end up suffering from .For example, a person suffering from OCD might end up checking multiple times sometimes hundreds of times thereby making a person frustrated leading to chronic stress. This prolonged stress can lead to clinical depression. People suffering from OCD might also suffer from fear of contamination, hoarding, ruminations and intrusive thoughts all of which give rise to major depressive disorders if continues to exist for a prolonged period of time.

Attention Deficit Hyperactivity Disorder(ADHD) [16] :

Attention deficit disorder (ADD) [30] is a neurological disorder that causes a range of behavior problems such as difficulty attending to instruction, focusing on schoolwork, keeping up with assignments, following instructions, completing tasks and social interaction. ADD is a term used for one of the presentations of attention deficit hyperactivity disorder (ADHD), as defined in the Diagnostic and Statistical Manual of Mental Disorders. It is officially, attention-deficit/hyperactivity disorder, predominantly inattentive presentation. Children with ADD without the hyperactivity component may appear to be bored or disinterested in classroom activities. They may be prone to daydreaming or forgetfulness, work at a slow pace and turn in incomplete work. As a result of which they get criticised on a regular basis which can bring about lethargic, pessimistic and depressive attitude.

\section{Chronic Allergy[19] :}

Allergy sufferers show symptoms like sneezing, sniffling, and red, itchy eyes. Recent studies show an association between seasonal allergies and clinical depression. While researchers can't say that allergies actually cause people to feel depressed, it does appear that allergy sufferers are more vulnerable to depression.

Most people who have allergies don't have depression, and most people who are depressed don't have allergies [20] says Paul S. Marshall, PhD, a clinical neurophysiologist in the department of psychiatry at the Hennepin County Medical Center in Minneapolis. "But I think it's accurate to characterize allergies as a risk factor for depression." Large-scale demographic studies suggest that allergy sufferers are roughly twice as likely to have depression as people without allergies. It has been seen through studies that adults with allergic rhinitis (hay fever) are twice as likely to have major depressive disorder in the previous 12 months. In a particular study it has been seen that kids who had hay fever at age 5 or 6 were twice as likely to experience major depression.

We have explained the allergy-depression connection in psychological terms, focusing primarily on the heavy emotional toll of chronic allergy symptoms. 


\section{Seasonal Affective Disorder [17]:}

Seasonal affective disorder (SAD) [20] is a type of depression that occurs due to changes in seasons - SAD begins and ends at about the same time every year.Treatment for SAD may include light therapy (phototherapy),

medications and psychotherapy.

\section{Symptoms:}

In most cases, seasonal affective disorder symptoms appear during late autumn or early winter and go away during the sunnier days of spring and summer. Less commonly, people with the opposite pattern have symptoms that begin in spring or summer. In either case, symptoms may start out mild and become more severe as the season progresses.

Signs and symptoms of SAD may include:

1. Feeling depressed most of the day, nearly every day.

2. Losing interest in activities you once enjoyed.

3. Always lacking in energy.

4. Having problems with sleeping.

5. Experiencing changes in your appetite or weight.

6. Feeling sluggish or agitated.

7. Having difficulty concentrating.

8. Feeling hopeless, worthless or guilty.

9. Having frequent thoughts of death or suicide.

All of the above mentioned tendencies, thoughts and feelings lead to depression.

\section{Causes:}

The specific cause of seasonal affective disorder remains unknown. Some factors that may come into play include:

1. Your biological clock (circadian rhythm). The reduced level of sunlight in fall and winter may cause winteronset SAD. This decrease in sunlight may disrupt your body's internal clock and lead to feelings of depression.

2. Serotonin levels. A drop in serotonin, a brain chemical (neurotransmitter) that affects mood, might play a role in SAD. Reduced sunlight can cause a drop in serotonin that may trigger depression.

3. Melatonin levels. The change in season can disrupt the balance of the body's level of melatonin, which plays a role in sleep patterns and mood.

\section{Complications:}

SAD can get really worse if not treated properly. It can lead to various types of severe complications such as social withdrawal, school or work problems, substance abuse, all of which lead to major depressive disorder leading to suicidal tendencies. Treatment can help prevent complications, especially if SAD is diagnosed and treated before symptoms get bad thereby reducing the risk of more people becoming victims of depression.

\section{Environmental Pollution [18]:}

Air pollution is the major cause for causing various kinds of stress and hence stress related disorders. Major depressive Disorder being the most prominent and common one. Stress is pernicious. Stress, especially chronic stress, can weaken the body's immune system. It can cause heart diseases, high blood pressure and a lot of other ailments. Stress is linked to leading causes of death from heart diseases to depression to suicidal tendencies. The origins of stress are mostly emotional. Mental health experts say money troubles, job conflicts, problems with relationships and major life changes are among the most common causes. However there is another, more surreptitious cause. Scientists have discovered that poor quality of air plays a role in increasing stress and chronic stress can lead to none other than Major Depressive Disorder. There is an acute link between exposure to ambient air pollution and depression in children. There is also a connection between air pollution and suicide. Researchers [31] at the Harvard School of Public Health recently studied the effects of fine particles, black carbon, nitrogen dioxide and other pollutants on stress. They analyzed data on 987 men and found perceived levels of stress rise when people are exposed to air pollution. The association was especially evident in colder weather and in relation to overall particle count levels.

It is mostly brain inflammation and hormonal activities are involved in the connection between pollution and stress. 
The most prone area of the human body that gets affected the most by pollution is the respiratory system leading to certain inflammations in the brain and severe hormonal disbalances that lead to clinical depression. One of the major ways to cope with stress and hence depression is to reduce exposure to pollution in all forms let alone air pollution. Managing stress is a key to keeping yourself hale and hearty. By recognizing stress early and taking action to control it, you will reduce your risk of a variety of illnesses depression being one of the most deadly and persistent ones.

\section{Experimental evaluation:}

To get the required result we had conducted few surveys for environmental and psychological disorders and studied about the mentioned genetic disorders from online database according to our research, in India. The surveys include the percentage of people suffering a particular disease in different groups, the most affected age group from a specific disorder, percentage of the role played by genetic disorders in depression.

\section{Studies for genetic disorders: \\ Study 1-}

In our first study we collected database from some open sources, naming them Group 1, Group 2 and Group 3. We used different data of people from various age groups having specific disorders and then compared all the data that shows how many people are suffering from that particular disease in different groups. By comparing the common age groups and then calculating all the data, it concluded with the result which shows that :

In Group 1, 2 and 3 the data of people having age from 13 to 60 has been studied respectively. The study was conducted for some specific disorders (Dysthymia, Bipolar Disorders, Schizophrenia, Schizoaffective Disorders, Autism Spectrum Disorder and Huntington's Disease); which shows that Dysthymia is mostly found among the people having age group of 19 to $30 \mathrm{yrs}$, Bipolar disorder is common among the people having age from 26 to $50 \mathrm{yrs}$, the people those belong to the age group between 30 to 40 yrs are most likely to suffer from Schizophrenia , Schizoaffective Disorders occurs mostly among the people who are 20 to $35 \mathrm{yrs}$ in age, the children having age from 9 months to 16 yrs are the most common victims of Autism Spectrum Disorder and Huntington's Disease is found mainly among the people from 30 to $50 y$ rs of age and worsen over a 10 to 25 year period.

\section{Study 2-}

In our second study we had evaluated different data of disorders and analyzed the major factor that is causing Major Depressive Disorders among the people. In the study we have found that majorly $60 \%$ of depression among the people is developing because of environmental factors and psychological factors while remaining $40 \%$ is the consequence of genetic factors. The role played by the genetic diseases, which we had studied, in causing depression are mentioned below:

16\% Dysthymia, 10\% Bipolar Disorder, 5\% Schizoaffective Disorder, 7\% Schizophrenia and 2\% other genetical issues.

Study 3-

Specifically, the study three includes the analyzed data of percentage of people suffering from the disorders, which we had studied for genetical causes, leading to Major Depressive Disorder. We evaluated the different data sets of people from India having those diseases and calculated the percentage of patients. The final result reveals the following outcome:

The percentage of people affected by given disorders in India are-

1. Schizophrenia- $7.4 \%$

2. Bipolar disorder- $7 \%$

3. Autism Spectrum Disorder-4.2\%

4. Schizoaffective Disorder $-0.3 \%$ to $1.1 \%$

5. Dysthymia- $14.5 \%$

6. Huntington's disease- $16.2 \%$. 

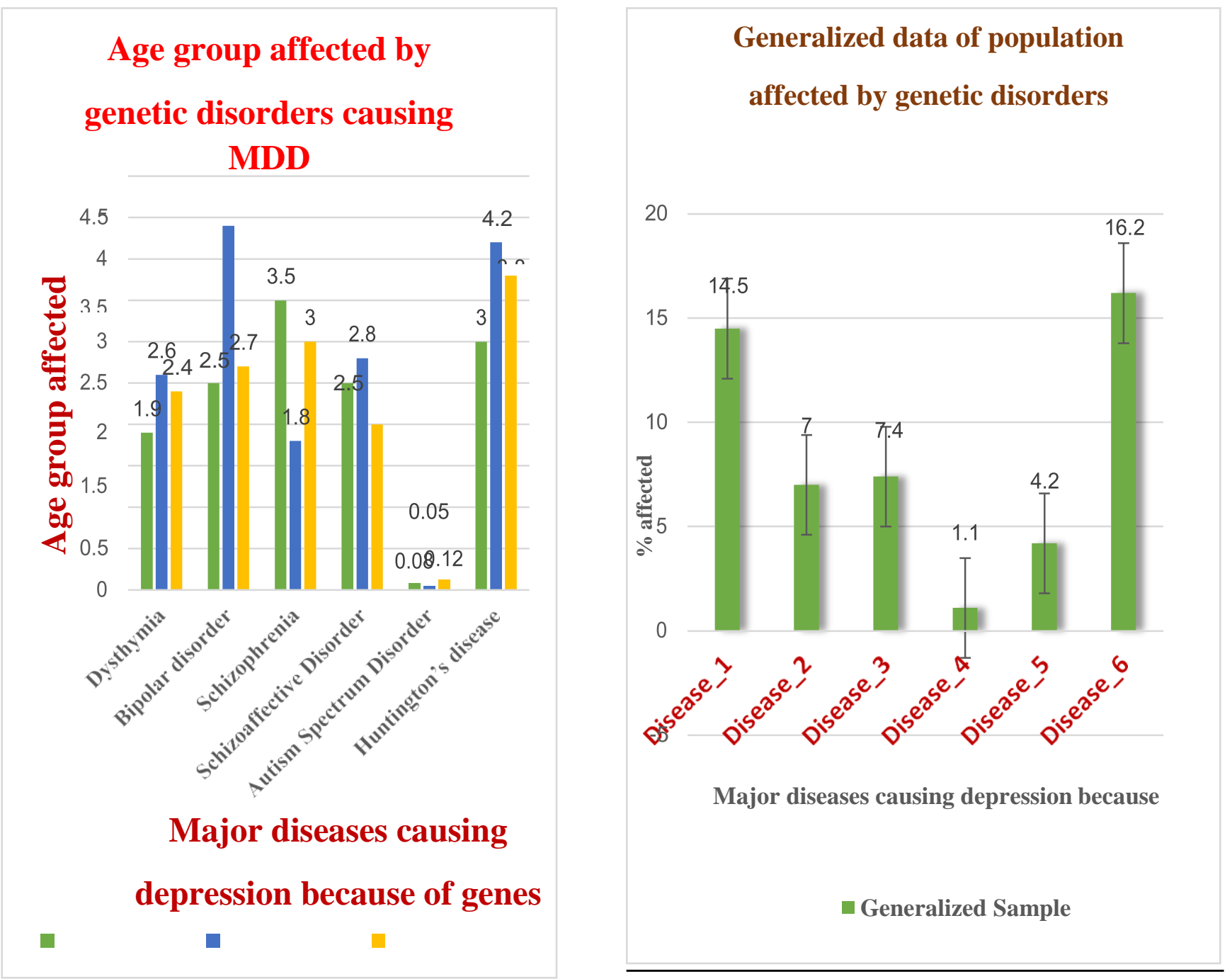

Figure 3:-Age group affected by genetic disorders causing MDD on Population_1, Population_2 and Population_3. Figure 4:-Generalized data of population affected by genetic disorders causing MDD in INDIA on Disease: $D_{n}:\{n=1: 6\}$.

\section{Studies for psychological disorders:}

We started with a simple survey of asking people to fill a google form that asked certain questions that helped us to deduce their personality types. There are 16 personality types broadly classified into :

\section{Analysts:}

1. Architect [ INTJ $(-\mathrm{A} /-\mathrm{T})]$

2. Logician $[\operatorname{INTP}(-\mathrm{A} /-\mathrm{T})]$

3. Commander $[\mathrm{ENTJ}(-\mathrm{A} /-\mathrm{T})]$

4. Debater $[\mathrm{ENTP}(-\mathrm{A} /-\mathrm{T})]$

\section{Diplomats:}

1. Advocate[INFJ(-A/-T)]

2. Mediator $[\mathrm{INFP}(-\mathrm{A} /-\mathrm{T})]$

3. Protagonists $[\mathrm{ENFJ}(-\mathrm{A} / \mathrm{-T})]$

4. Campaigner $[\mathrm{ENFP}(-\mathrm{A} /-\mathrm{T})]$

\section{Sentinels:}

1. Logistician [ISIJ(-A/-T)] 
2. Defender [ISFJ(-A/-T)]

3. Executive $[\mathrm{ESTJ}(-\mathrm{A} / \mathrm{-T})]$

4. Consul $[\mathrm{ESFJ}(-\mathrm{A} /-\mathrm{T})]$

\section{Explorer:}

1. Virtuoso [ISTP(-A/-T)]

2. Adventurer [ISFP(-A/-T)]

3. Entrepreneur $[\mathrm{ESTP}(-\mathrm{A} / \mathrm{-T})]$

4. Entertainer $[\mathrm{ESFP}(-\mathrm{A} / \mathrm{-T})]$

All of these personality types have their own weaknesses and these weaknesses help us to let us know whether they will suffer from major depressive disorder or are already suffering from it or not .

We conducted the survey in three groups in three groups of people, one that consisted of a majority of youngsters( mostly young adults from age group of 16-22); second group consisting of middle aged people(25-35 yrs) and the third group having a majority of people within the age group of 40-60yrs). After doing a detailed analysis of the various personality types we found out that every personality type has its own set of weaknesses. Some of these weaknesses or flaws in their personality traits have the tendencies of making the person pessimistic, frustrated, stressed and hence a victim of Major Depressive Disorder. From our survey we found out some of the most common personality traits that can lead to MDD. People suffering from insecurities from a prolonged period of time tend to gradually become overly depressed and lose their vitality in life. Some of them might even develop suicidal tendencies in the future. Certain personality types have shown over-analytical and over empathetic characteristics. Both of which are not very good for patients of clinical depression.

The antipathy to rules and tendency to over analyse and be judgmental, even arrogant, ads up to a personality type that is often clueless in dating thereby living them frustrated and henceforth depressed. This was found to be a major problem amongst teenagers and adults. Unrelenting imagination leads to disappointments which if becomes a regular thing leads to chronic stress. From our survey we found out that chronic stress is one of the most common reason behind depression. Despite the advent of science and technology almost $75 \%$ of all the 3 groups were found out to be introverts. It was also found that a needy attitude prevailed amongst $45 \%$. This needy attitude or feeling too introverted all the time can be a prominent cause for depression.

Condescending: attempts at connecting with others or often worse than INTP's withdrawal. It was found from the questions asked in the survey that people with the INTP personality type take pride in their knowledge and rationalise, and enjoy sharing their ideas, however in trying to explain how they got from A to B to Z, they can get frustrated very easily, sometimes simplifying things to the point of insult as they struggle to gauge their conversation partners' prospective. The ultimate insult comes as they give up with a dismissive "Nevermind". This leads to tremendous amount of dissatisfaction and unhappiness amongst these personality types, filling them up with lots of pessimism. This can lead to Major Depressive Disorders. The self-loathing nature found in some can also lead to MDD. The survey indicates that INFPs sometimes see themselves as selfish but only because they want to give so much more than they are able to.This becomes a self-fulfilling prophecy, as they try to commit to a chosen cause or person, forgetting to take care of the needs of others in their lives, especially themselves which can lead to Midlife Crisis later in their lives.This too altruistic nature is a major cause leading to clinical depression.

Extremely condemning nature can lead to a person being highly depressive in nature too. Our survey also found that ISFJ personality types show Repression of their feelings to a great extent where they internalize their feelings a great deal. This lack of healthy emotional expression can lead to a lot of stress and frustration that gradually builds up depression. Last but not the least another major reason why many extroverts have been found to suffer from depression is Hypochondria. 


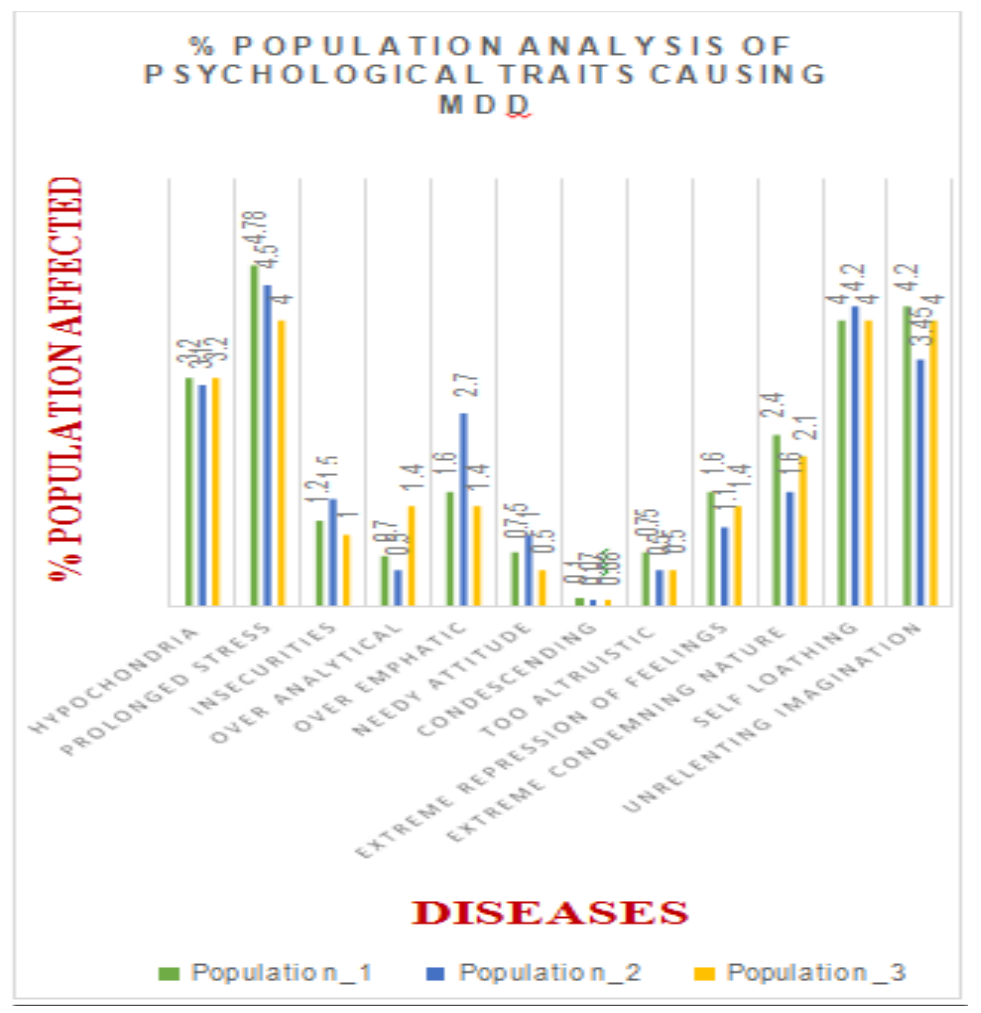

\section{Studies for Environmental disorders :}

We conducted another survey that concluded from a basic set of questions whether a person suffers from OCD or ADD or chronic allergies or SAD. Almost 85\% were found to suffer from ADD .25 to $30 \%$ from allergies although chronic allergies were found only among 8 to $10 \%$ On the other hand $5 \%$ were found to suffer from SADs. $20 \%$ suffer from OCD.

\section{Conclusion and future works:}

The above studies dealing the genetic factors of several diseases which are provoking Major Depressive Disorder shows that the $40 \%$ of the depression is caused because of certain genetic disorders. Every disease which we had studied,i.e. Schizophrenia, Bipolar disorder, Autism Spectrum Disorder, Schizoaffective Disorder, Dysthymia, Huntington's disease, have a certain age group of people which are getting affected the most by each disease respectively, in India. There are several reasons why a person suffers from depression. It is all in the mind and $90 \%$ cause of depression can be attributed to psychological reasons and personality flaws.

Every person has a different personality type and hence classifying them all into one category would be unfair and incorrect. However in order to reach to a conclusion we might say that introverts tend to suffer from greater depression than extroverts or ambiverts. One of the most common reasons why people tend to suffer from depression is chronic stress and frustration given rise by their various personality flaws. From the conducted survey and detailed analysis of it we finally come to the conclusion that one of the most prominent reasons behind depression is ADD and pollution. Further research await to be done on the concepts of personality analysis and depression caused due to homosexuality and other queer sexual orientations, food habits, lifestyles, Oedipus complex and Post rape trauma. There are various fields left to do research on in the future such as psychotherapy for people who suffer from personality flaws, LGBTQ community and 
their demeanor. A Research on Auxiliary Breast Tissue and also on Cystic Fibrosis.

\section{Acknowledgement:-}

We would like to express our special thanks of gratitude to our professors of SRM Research Day who gave the golden opportunity to write this paper on Genetic Engineering and Psychology which also helped us to learn lots of new ideas and concepts in our respective fields of interests.

This research was supported and encouraged by Next Tech Lab to a great extent. Joining Next Tech Lab was one of the best things that happened to us here in SRMIST apart from the various other benefits provided to us by this prestigious institution. We have gained knowledge and learnt new things. Our seniors in the Lab have been very supportive; especially there conducted workshop and classes were very useful. They supported our ideas all the time. We would like to thank our syndicate Vaishnavi Balaji (syndicate of Healthcare).

We are thankful to our friends who assisted us in conducting the survey. We are also grateful to Sree Harsha Nelaturu (Head of Next Tech Lab) for his assistance in moderating this paper and in that line improving the manuscript significantly.

\section{References:-}

1. CRY2 Genetic Variants Associate with Dysthymia by Leena Kovanen, Mari Kaunisto, Kati Donner, Sirkku T. Saarikoski, Timo Partonen

2. Schizophrenia by National Alliance Of Mental Hostel WebMD

3. Huntington Disease in Asia by Miao Xu and Zhi-Ying Wu Huntington's Disease Society of America

4. Healthline: Is Depression Genetic? Medically reviewed by Timothy J. Legg, PhD, CRNP on October 10, 2016 - Written by Stephanie Faris.

5. American Council On Science and Health: Is There A Depression Gene? By Julianna LeMieux - April 6, 2017

6. Genetic link for depression found: Analysis by Bazian Edited by NHS Website

7. American Psychiatric Association

8. Gulf Bend Center (Article on Biology of depression)

9. Gene-Environment Interaction and the Genetics of Depression by Klaus Peter Lesch

10. National Center for Biotechnology Information

11.National Institute Of Mental Health: Obsessive-Compulsive Disorder

12. Types of OCD by ocduk.org

13. Defining Attention Deficit Disorder (ADD) Without Hyperactivity; Symptoms and Differences by Ann Logsdon

14. Seasonal affective disorder (SAD) by Mayo Clinic

15. Air pollution, stress and your health by Air Quality Life.

16. Are Allergies Making You Depressed? By David Freeman

17. Effects of Seasonal Allergic

18. Rhinitis on Fatigue Levels and Mood by Marshall, Paul S. PhD; O'Hara, Christine RN, and; Steinberg, Paul MD

19. CRY2 Genetic Variants Associate with Dysthymia by Leena Kovanen, Mari Kaunisto, Kati Donner, Sirkku T. Saarikoski, Timo Partonen

20. Genomics and Pharmacogenomics of Brain Disorders by Cacabelos,

21.Ramon; Martinez-Bouza, Rocio; Carlos Carril, Juan; Fernandez-Novoa, Lucia; Lombardi, Valter; Carrera,Ivan; Corzo, Lola; McKay, Adam

22. Genome Scan Meta-Analysis of Schizophrenia and Bipolar Disorder, Part II:

23. Schizophrenia by Cathryn M. Lewis, Douglas F. Levinson, Lesley H. Wise, Lynn E. DeLisi, Richard E. Straub, Iiris Hovatta, Nigel M. Williams, Sibylle G. Schwab, Ann E. Pulver, Stephen V. Faraone, Linda M. Brzustowicz, Charles A. Kaufmann, David L. Garver, Hugh

24. M. D. Gurling, Eva Lindholm, 19 Hilary Coon, Hans W. Moises, William Byerley, Sarah H. Shaw, Andrea Mesen, Robin Sherrington, F. Anthony O’Neill, Dermot Walsh, Kenneth S. Kendler, Jesper Ekelund, Tiina Paunio, Jouko Lo"nnqvist, Leena Peltonen, Michael C. O’Donovan, Michael J. Owen, Dieter B. Wildenauer, Wolfgang Maier, Gerald Nestadt, Jean-Louis Blouin, Stylianos E. Antonarakis, Bryan J. Mowry, Jeremy M. Silverman, Raymond R. Crowe, C. Robert Cloninger, MingT. Tsuang, Dolores Malaspina, Jill M.

25. Harkavy-Friedman, Dragan M. Svrakic, Anne S. Bassett, Jennifer Holcomb, Gursharan Kalsi, Andrew 
McQuillin, Jon Brynjolfson, Thordur Sigmundsson, Hannes Petursson, Elena Jazin, Tomas Zoe"ga, and Tomas Helgason

26. A review of gene linkage, association and expression studies in autism and an assessment of convergent evidence by Mao Sheng Yang, Michael Gill.

The Genetics of Schizophrenia byb Mohd Razali Salleh

1. Hippocampal total volume, cell numbers, and astrocytes in major depressive disorder by Cobb, Justin A. The University of Mississippi Medical Center, ProQuest Dissertations Publishing, 2012.

2. Nonsynonymous Variation

in NKPDIIncreases Depressive Symptoms in European Populations by Najaf Amin,Nadezhda M. Belonogova,Olivera Jovanova,Rutger W.W. Brouwer,Jeroen G.J. van Rooij,Mirjam C.G.N. van den Hout,Gulnara

R. Svishcheva,Robert Kraaij,Irina V. Zorkoltseva,Anatoly V. Kirichenko,Albert Hofman,André G. Uitterlinden et al.

3. Personality Determinants of Mass Media Preferences by Philip Anast, Ph.D.

4. Symptoms of Obsessive-Compulsive Disorder by James F. Leckman, M.D., Dorothy E. Grice, M.D., James Boardman, M.D., Heping Zhang, Ph.D., Amy Vitale, B.A., Colin Bondi, M.A., John Alsobrook, Ph.D., Bradley S. Peterson, M.D., Donald J. Cohen, M.D., Steven A. Rasmussen, M.D., Wayne K. Goodman, M.D., Christopher J. McDougle, M.D., and David L. Pauls, Ph.D.

5. Attention-Deficit Hyperactivity Disorder by Russell A. Barkley

6. A Framework for Examining Social Stress and Susceptibility to Air Pollution in Respiratory

Health by Jane E. Clougherty

and Laura D. Kubzansky

7. The World Health Report 2001: Mental Health : New Understanding, New Hope By World Health Organization

8. Fre

ud

scientifi

cally

reapprai

sed:

Testing

the

theories

and

therapy

S Fisher, RP Greenberg - New York, 1996

9. $\quad$ Erik

Erikson's theory

of psychosocial

development

and vocational

behavior.

PH Munley - Journal of Counseling

Psychology, 1975 